\title{
Evaluating the impacts of the multi-microgrid concept using multicriteria decision aid
}

\author{
J. Vasiljevska*, J.A. Peças Lopes, M.A. Matos \\ Faculty of Electrical and Computer Engineering, University of Porto, Instituto de Engenharia de Sistemas e Computadores do Porto (INESCPorto), 4200-465 Porto, Portugal
}

\section{A R T I C L E I N F O}

\section{Article history:}

Received 2 May 2011

Received in revised form 5 April 2012

Accepted 20 April 2012

\section{Keywords:}

Microgrid

Multi-microgrid

Multiattribute assessment

Trade-offs

Decision-aid

\begin{abstract}
A B S T R A C T
Exploiting the benefit that one can get from large scale integration of microgeneration $(\mu \mathrm{G})$ requires the adoption of different control strategies at different distribution network levels. In that sense, MicroGrid (MG) and Multi MicroGrids (MMG) concepts may be seen as a way to facilitate integration of higher levels of $\mu \mathrm{G}$ into the system. This paper describes new control and management functionalities used to take profit from the presence of microgeneration and active loads under MG and MMG concepts. MultiCriteria Decision Aid (MCDA) techniques are used to capture decision maker's preferences in evaluating the impact of MG and MMG concepts deployment and help selecting the best development strategy.
\end{abstract}

(C) 2012 Elsevier B.V. All rights reserved.

\section{Introduction}

The need to reduce $\mathrm{CO}_{2}$ emissions to cope with the environmental changes and the foreseen future scarcity of fossil fuels are leading to the development of policies that involve two synergetic paths: (a) full exploitation of renewable power resources to produce electricity and (b) more efficient use of electric energy. Since renewable power resources are geographically distributed and are characterized by some variability, it is necessary to develop flexible solutions to manage the electric distribution network, namely when integrating large amounts of small and $\mu G$ units that exploit different sources. Such solutions regard adoption of adequate mechanism of control and management of the $\mu \mathrm{G}$ units together with local loads in order to facilitate full integration of $\mu \mathrm{G}$ into the distribution grids. Thus, the Low Voltage (LV) grids should become active and can then be considered as a kind of a cell of a complex body. This cell is defined as a microgrid [1], and assumed to be a key player in the future operation of distribution grids.

If now one considers that Medium Voltage (MV) grids will have several LV MGs, together with several Distributed Generation (DG) units directly connected to the grid, in conjunction with MV controllable loads, then we are introducing the MMG concept [2]. A specific MMG control and management architecture will link the

\footnotetext{
* Corresponding author. Tel.: +351 222094 198; fax: +351222094 150.

E-mail addresses: vjulija@inescporto.pt (J. Vasiljevska), jpl@fe.up.pt (J.A. Peças Lopes), mmatos@inescporto.pt (M.A. Matos).
}

DG and $\mu \mathrm{G}$ sources with active demand, requiring a development of new control functionalities capable to exploit the existing flexibility of local generation and load. These functionalities are expected to deal with the greater complexity that increased presence of $\mu G$ and DG will bring to the system [3]. A hierarchical control approach is used in [4], where market based functionality is developed, responsible for the optimization of the MG operation. The economic, technical and environmental impacts may then be evaluated at LV and MV network level, referring to a set of costs and benefits, if one adheres to the MMG concept [5].

This paper proposes new functionalities able to deal with stressed operating conditions (overloads and excessive voltage drops) that may arise as a consequence of grid reconfiguration or due to natural load growth. Such control functionalities are assumed to be used by the Distribution System Operator (DSO) while facing growing potential of $\mu \mathrm{G}$, together with active loads, under massive MGs deployment.

The benefits due to the exploitation of this approach can be attributed to several stakeholders involved in the MMG concept deployment. By analysing the impacts that the MMG provokes on the distribution network, it will be possible to derive new regulatory approaches, leading to the creation of proper incentive mechanisms for the DSO, $\mu \mathrm{G}$ owners and loads to adhere to this concept. MCDA techniques can then be used to capture the DSO preferences when analysing the potential benefits and costs regarding the MMG concept deployment, and helping, in this way, in defining the best development strategy in a presence of large $\mu \mathrm{G}$ penetration and demand side integration levels. 

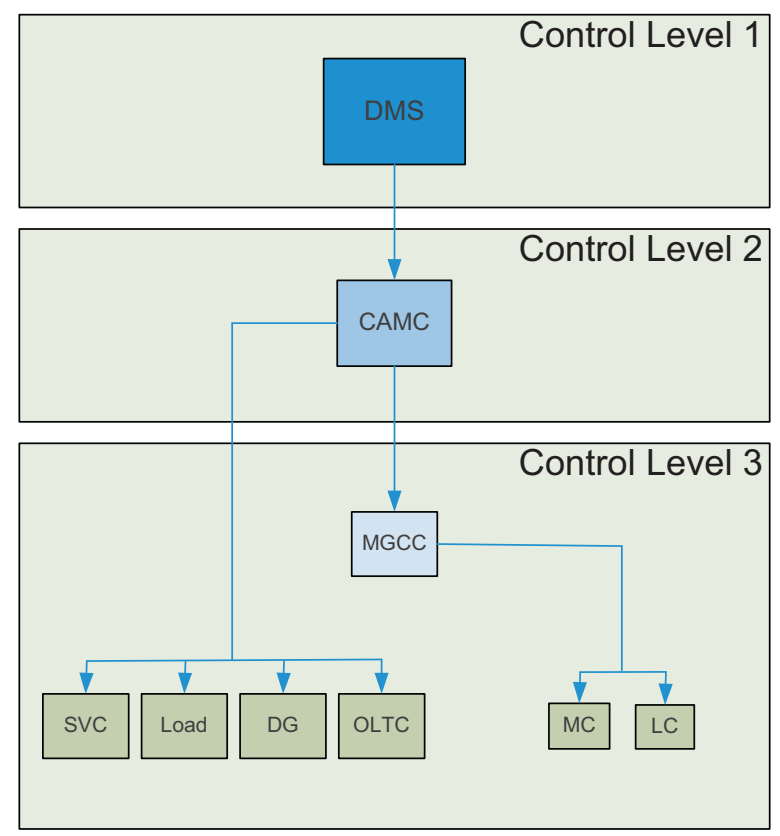

Fig. 1. Hierarchical control scheme.

\section{Multi-microgrid control and management structure}

The possibility of actively managing the network through the MG concept is a key aspect in the development of advanced control functionalities for active distribution grids. For this purpose a hierarchical control scheme architecture comprising three different control levels (Fig. 1), has been assumed for a MMG operation [1]: (1) Local Microsource Controllers (MC) and Load Controllers (LC); (2) MicroGrid Central Controller (MGCC), housed at the MV/LV secondary substations; (3) Central Autonomous Management Controller (CAMC), installed at the HV/MV substation level, and owned by the DSO.

Moreover, two types of $\mu G$ units are considered under this approach, controllable $\mu \mathrm{G}(\mathrm{C}-\mu \mathrm{G})$ i.e. fuel consuming and noncontrollable $(\mathrm{NC}-\mu \mathrm{G})$ i.e. renewable power resources - PV and micro wind generation. Loads can be also considered as controllable loads (CLs) and non-controllable (critical).

Under this approach, for each predefined time interval (e.g. $1 \mathrm{~h}$ ), a MG optimization procedure (e.g. using a local retail market) is performed at MGCC level [4]. This retail market is assumed to be operated by an independent network operator acting in favour of optimizing the MG operation, taking into account electricity market prices, MG demand and controllable $\mu \mathrm{G}$ bids. Such trading mechanism grants the $\mathrm{C}-\mu \mathrm{G}$ units with the possibility of selling at higher (than wholesale market) prices while the MG consumers obtain better electricity price due to avoided grid charges for the $\mu \mathrm{G}$ produced and consumed locally [6].

The management of the MV multi-microgrid is performed through a new intermediate level control entity - the Central Autonomous Management Controller (CAMC). CAMC may be seen as a Distribution Management System (DMS) application which is responsible for part of the MV network below a particular HV/MV substation [2]. A proper MMG operation requires adoption of decentralized control strategy, whereas the decision making still holds a hierarchical structure (Fig. 1) [2]. However, this structure still allows an autonomous operation during islanded operating mode of each MG within the MMG.

The CAMC, serving as an interface for the DMS, communicates with other local controllers, such as MGCC and DG units or loads connected directly to the MV network in order to manage the MMG in both HV grid-connected operating mode and emergency operating mode [2]. However, due to the concept defined in this work, the distribution network is assumed to operate in two operating modes when connected to the HV grid: normal operating mode, where thermal branch and voltage levels are within the allowable limits and stressed operating mode, involving a violation of any steady state technical constraint, that may lead to potentially high branch overloads and/or voltage drops.

\subsection{Load and $\mu G$ management strategies under CAMC}

If stressed operating conditions are detected at the MV grid level, $\mu \mathrm{G}$ and loads will be controlled from the CAMC through broadcasted control signals. Controllable LV loads, not being shifted under MGCC control level, are assumed to accept curtailment requests under predefined curtailment contracts between the DSO and the MMG consumers (through the respective MGCCs). Each curtailment contract determines the upper limit of the curtailable load, the cost of curtailing, time intervals and time limitations for load curtailing.

The CLs seen from the MGCC level, at each LV bus, may reflect different prices for load curtailment at each time interval in a day. However, the CAMC is not interested in knowing which load, within a specific MG, will be curtailed, as long as the curtailment cost is equal for all the CLs inside a particular MG. The CAMC just "sees" the available aggregated CL at each MV/LV transformer level. Moreover, $C-\mu \mathrm{G}$ units that have still room for being dispatched from the CAMC level are considered available to be contracted, under special contractual agreements between the DSO and the controllable $\mu \mathrm{G}$ units' owners within each MG (via the respective MGCCs). Each contractual agreement determines the lower and upper limit of each $\mathrm{C}-\mu \mathrm{G}$ unit and its operational cost [4].

\subsection{MMG control and management}

A new functionality to be installed at the CAMC level is envisaged in order to deal with the stressed MV operating conditions. In the stressed operating mode this functionality controls the amount of available $C-\mu G$ to be produced and/or CL to be curtailed, subject to predefined contractual agreements and/or curtailment contracts in a given time interval. This requires the CAMC to communicate permanently with the available $C-\mu G$ sources and $C L s$ through each MGCC in order to receive information about the $C-\mu G$ production availability and/or CL willing to be contracted for load curtailment.

The proposed control and management approach may bring several technical and economic benefits to the DSO for both operating modes, namely: (1) LV and MV active losses reduction; (2) emissions' reduction caused by active losses reduction; (3) reduction in the emissions due to displacement of central thermal generation units by $\mu \mathrm{G}$ production with no emissions or less emissions; (4) network investment deferral (measured in years); (5) improved quality of service, regarding both continuity of supply and voltage quality.

The operation management problem for stressed conditions for a three phase balanced MV grid can be formulated as an optimization procedure, involving the minimization of operation costs, performed at CAMC level, for each time interval (for instance, $1 \mathrm{~h}$ ) of the foreseen/actual stressed operating conditions:

$\min C_{\text {operation }}=\min \left\{\sum_{i=1}^{N} C_{1 i}+\sum_{j=1}^{L} C_{2 j}+C \cdot P_{\text {losses }}\right\}$

s.t.

$g(\boldsymbol{P}, \boldsymbol{Q}, \boldsymbol{U}, \boldsymbol{\theta})=0$ 
$\left|I_{i j}\right| \leq\left|I_{i j}^{\max }\right|$

$\left|U_{i}^{\max }\right| \geq\left|U_{i}\right| \geq\left|U_{i}^{\min }\right|$

$P_{G i}^{\min } \leq P_{G i} \leq P_{G i}^{\max }$

$0 \leq P_{L j}^{\prime} \leq P_{L j}$

$P_{\text {losses }}=\sum_{i=1}^{n} \sum_{j=n+1}^{m} R \cdot\left|I_{i j}\right|^{2}$

$\underline{I_{i j}}=\frac{\left(\underline{U_{i}}-\underline{U_{j}}\right)}{\underline{Z_{i j}}}$

$C_{1 i}=a_{i} \cdot P_{G i}+b_{i}$

$C_{2 j}=c_{j} \cdot P_{L j}^{\prime}$

In this formulation the load flow problem (2) is solved in p.u., while for the remaining equations real values are used. The control variables are $P_{G i}[\mathrm{~kW}]$ and $P_{L j}^{\prime}[\mathrm{kW}]$, which correspond to the C$\mu \mathrm{G}$ dispatched at bus $i$ and CL curtailed at bus $j$. Eq. (2) presents the active and reactive power flow equations, where $\boldsymbol{P}$ and $\boldsymbol{Q}$ are vectors of the foreseen/present active and reactive power injections at each bus (in p.u.) for the study period. The active power injection at each bus $i$ (except the reference), $P_{i}$ is given in (11):

$P_{i}=\left(P_{G i(n c)}+P_{G i}\right)-\left(P_{L i(n c)}+P_{L i}-P_{L i}^{\prime}\right)$

where $P_{G i(n c)}$ is the non-controllable generation dispatched once its primary source is available. $P_{L i(n c)}$ is the non-controllable part of the total demand at each MV bus $i$. The power injection at the reference bus equals the power coming from the upstream network.

Under this formulation, $P_{G i}$ and $P_{L i}^{\prime}$ account for the reduction of the active power injection and resolution of the problem of overloads or excessive voltage drops. Generally, $P_{G i}$ may be dispatched or $P_{L i}^{\prime}$ shifted under MGCC in the local retail market due to high wholesale electricity prices and account for reduction of the active power injection at the MV bus $i$. The DSO can then get the benefits from partial or total resolution of the technical constraint violation without having to pay for it. $\boldsymbol{U}$ and $\boldsymbol{\theta}$ are vectors of bus voltage magnitude and phase angle, respectively, resulting from the solution of the power flow for the specified operating conditions. Inequality constraints (3) and (4) stand for the technical constraints, in terms of branch current and voltage limits, whereas (5) and (6) present the upper and lower manageable limits of each C- $\mu \mathrm{G}$ unit and CL subject to contractual agreements and curtailment contracts between the DSO and the $\mu \mathrm{G}$ owner/MMG consumer, respectively. Eq. (7) describes the total active MV network losses, whereas (8) stands for the current in each branch $I_{i j}$, with $U_{i}$ and $U_{j}$ being the $i$ and $j$ bus voltages, respectively, and $Z_{i j}$ the $\overline{i j}$ branch impedance. The parameters $a_{i}[€ / \mathrm{kWh}]$ and $b_{i}[€ \overline{/ \mathrm{h}}]$ in (9) present the cost coefficients of each $C-\mu \mathrm{G}$ unit, and $c_{j}$ in (10) stands for the unitary cost per $\mathrm{kWh}$ of CL curtailed. $C[€ / \mathrm{kWh}]$ in (1), is the electricity price for the respective time interval.

The MMG optimization strategy described before was solved in MATLAB environment, using a Sequential Quadratic Programming (SQP) routine based on quasi-Newton method [7]. Following the solution of this problem, $\mu \mathrm{G}$ set-points or/and load curtailment commands have been sent to each MGCC locally.

An approach which identifies potential costs and benefits should be central to the MMG impact evaluation by addressing the real benefits (and costs) that $\mu \mathrm{G}$ under the concept MMG can bring to the distribution networks in order to find out the right incentives to encourage the DSO, $\mu \mathrm{G}$ owners and consumers to be involved in the MMG concept deployment. The impact that the MMG deployment may have on the distribution network is treated as MCDA problem. Thus, a careful choice of relevant attributes for each criterion should be made addressing the impact of the large scale integration of $\mu \mathrm{G}$ and demand side integration under the MMG concept.

\subsection{MMG installation and operation cost}

The annualized cost of setting up the MMG includes the cost of the CAMC functionality related to the MMG optimization procedure, the MGCC cost in each MG, MC and LC cost for each $\mu \mathrm{G}$ units and consumer, respectively, in each MG.

The communication infrastructure needed for the development of the MG and MMG concepts is supposed to exploit the same automatic meter reading infrastructure adopted for remote advanced metering (smart metering) [8]. Also in this approach the communication requirements have been designed to support the additional needs of communication that the MG concept requires. Therefore, this cost is not considered in this analysis.

The annualized cost of the $\mathrm{C}-\mu \mathrm{G}$ units dispatched at CAMC level together with the annualized curtailment cost of the CL curtailed in stressed MV operating periods stands for the MMG operation cost, incurred by the DSO. The total MMG cost (installation and operation cost), assigned as a negative benefit, presents the first criterion $\left(C_{1}\right)$ in evaluating the impact that MMG concept deployment may have on the distribution network.

In the process of evaluation of benefits due to MMG concept deployment, the consumers within the MMG enjoys some benefits as well, such as, quality of service improvement, electricity bill reduction [9]. A proper evaluation of these benefits may lead to higher participation of the MMG consumers in the MMG installation cost and potentially to different final conclusions. Currently, we assume a simple sharing mechanism, where the MMG installation cost is equally distributed between the DSO and the MMG consumers.

\subsection{Benefits assessment}

Controllable $\mu \mathrm{G}$ units' dispatch and CL shedding under MGCC and/or CAMC in normal and stressed MV operating hours account for a set of benefits for the DSO, as mentioned in Section 2.2. All these benefits reported above need to be annualized to the first year when actual network investment, due to natural load growth, is required.

The investment deferral is referred as a second criterion $\left(C_{2}\right)$ in the evaluation of the MMG impact on the distribution network. This criterion will be evaluated for the worst MV branch or (MV/LV transformer) due to overloads or voltage drop for the peak hour(s) [10] in the year where network reinforcement is needed [11]. For this purpose, two typical load and generation profiles are used for every day due to two annual seasons (summer and winter). In typical rural networks critical voltage drops are expected due to the length of the wires, whereas in typical urban networks branch overloads are most likely to appear.

Moreover, MMG concept adoption may minimize the MMG operation cost as a result of distribution network losses reduction (benefit attributed to the DSO). In addition, the loss reduction due to MMG deployment can be interpreted as avoided cost due to avoided active losses, indicating the third criterion $\left(C_{3}\right)$ in the evaluation of the MMG concept solutions.

Relatively low global $\mu \mathrm{G}$ penetration level is assumed in our study so that only the most expensive units of the system are expected to be affected (the so-called marginal units). Therefore, only the emissions of these units should be taken into account, when evaluating the environmental impact of $\mu \mathrm{G}$ in form of $\mathrm{MG}$ and MMG (being the fourth criterion $-C_{4}$ ). However, such information is not as easily available and requires simulation of the power system daily schedule [12]. In addition, the C- $\mu \mathrm{G}$ units within the 


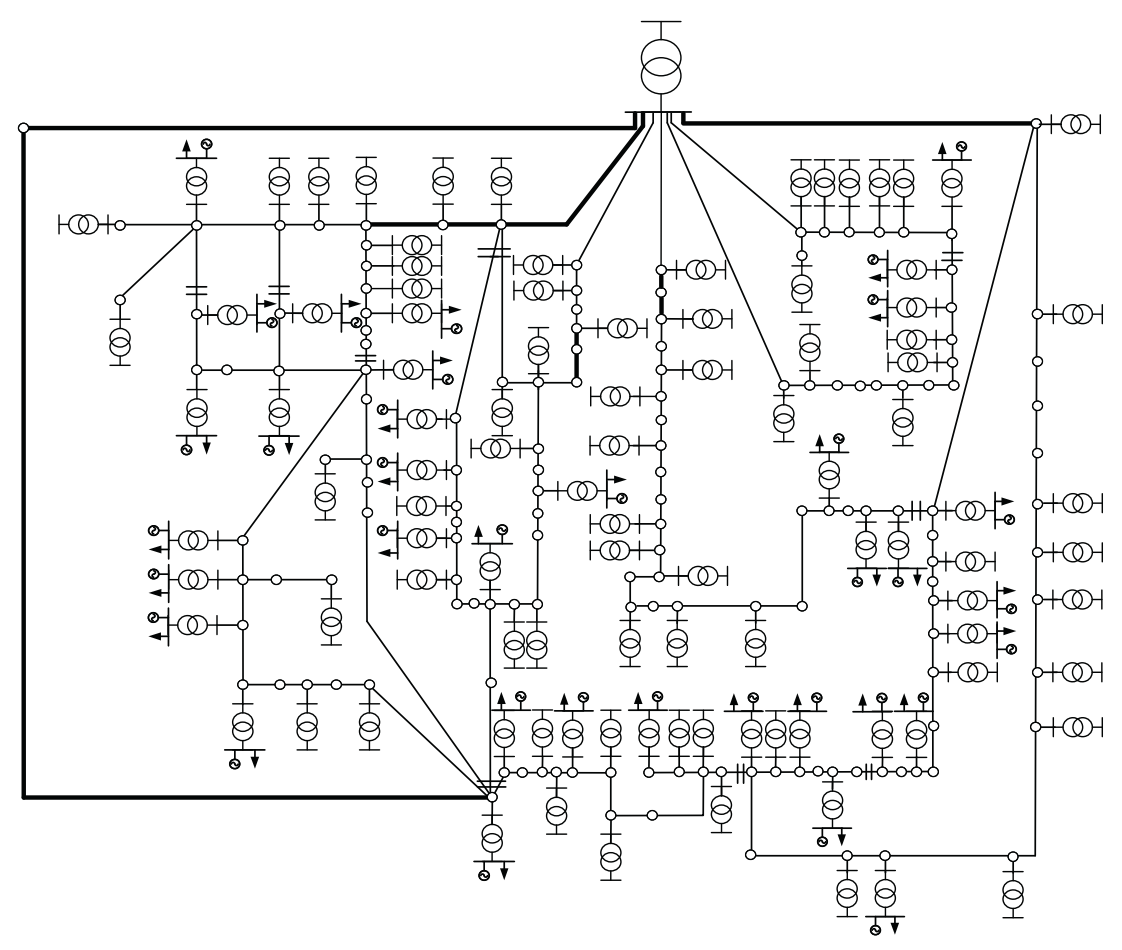

Fig. 2. $15 \mathrm{kV}$ urban MV network.

MMG have their own emissions, which should be considered in evaluating their environmental impact.

Furthermore, the microgrid's ability to work as a controllable entity with the possibility of functioning isolated from the main grid may improve the network reliability. This reliability improvement can be evaluated through the Non Distributed Energy (NDE). Depending on the regulation in force, an increase in DSO's revenue can be obtained as a result of this reliability increase. Assuming that all the $\mu \mathrm{G}$ is consumed, the value of the annual avoided NDE due to MMG operation and control may be estimated by:

$V_{\mathrm{NDE}}^{\mathrm{DSO}}=\sum_{\mathrm{MG}} \sum_{G} \lambda_{u p} \cdot\left(r_{u p}-P_{M} \cdot T_{a}\right) \cdot\left(\frac{W_{\mu \mathrm{G}(n c)}}{T}+P_{\mu \mathrm{G}(c)}\right)$

Eq. (12) assumes that in average the upstream network will fail $\lambda_{u p}$ times a year during $r_{u p}$ hours, leading to a certain amount of NDE. Isolated operation during these periods feeds a load of $\left(W_{\mu \mathrm{G}(n c)} / T+P_{\mu \mathrm{G}(c)}\right)$, resulting in avoided NDE. $W_{\mu \mathrm{G}(n c)} / T$ and $P_{\mu \mathrm{G}(c)}$ are the annual average power of the NC- $\mu \mathrm{G}$ and the capacity of the $C-\mu G$, within each MG, respectively. $P_{M}$ refers to the possibility of the MG to fail the isolating process and $T_{a}$ is the mean time to restore the MG after a complete shutdown, assuming that $T_{a} \ll r_{u p}$. At this stage, it is relevant to mention that a set of MGs together with Distribution Generation (DG) units directly connected to the MV network may account for the capability of MMG to operate isolated from the High Voltage (HV) network. However, since in our study no DG units are assumed to be connected directly to the MV network, we assume that the reliability improvement comes only from the ability of each MG to operate isolated from the MV network, feeding its internal consumers [13].

The avoided NDE due to the $\mu \mathrm{G}$ units inside the MMG, assigned as an indirect benefit to the DSO, presents the fifth criterion $\left(C_{5}\right)$ in the evaluation process of MMG adoption.

\section{Case study}

The impact assessment of the approach described before is performed at LV and MV typical Portuguese distribution networks, assuming different control strategies. In order to account for the benefits inside the MGs, either as a result of the local optimization procedure (performed at MG level) or due to the activation of the new control functionality performed under CAMC, LV networks are analysed (starting from each MV bus where a MG is assumed to be placed). In the former case, for each time interval during the period studied, load flow runs inside each MG. In the latter case, when control measures suggested by the CAMC are performed, a load flow runs also in each MG, so as to identify the level of energy losses reduction inside each $\mathrm{MG}$.

Fig. 2 illustrates a $15 \mathrm{kV}$ urban distribution network, with 35 MGs placed and the 10 most loaded lines denoted in bold. The peak load consumption is $25,908 \mathrm{~kW}$, at hour 21 in year 0 with a total daily energy demand of $497,184 \mathrm{kWh}$. With a total of 153 nodes and 163 branches, this network is operated in a radial form when in normal network operating mode. All $\mu \mathrm{G}$ units produce active power at unity power factor and the power factor of all loads is equal to 0.93 lagging. Since the branch lengths are generally short, the main operational problems in this network are related with the violation of the branch load limits.

Values of $0.23 € / \mathrm{kWh}, 0.24 € / \mathrm{kWh}$ and $0.25 € / \mathrm{kWh}$ (2-3 times higher than the value of electricity cost for the residential consumer in Portugal [14]), have been randomly assigned as load curtailment cost to different aggregated loads at each MV/LV transformer level (where a MG is assumed to be placed), whereas a value of $0.2 € / \mathrm{kW}$ h has been assumed for the unitary operation cost of the fuel-consuming $\mu \mathrm{G}$ units within each MG [15].

For purpose of the simulations performed in this research, a maximum of 4 curtailments within a day is assumed for each MG controllable load with maximum duration of 3 consecutive hours. However, these parameters may have different values, depending on the type of contract between the DSO and the consumers, i.e. the type of available $\mathrm{CL}$, number and time duration of the load interruptions.

For this study it was assumed that the cost for putting in place the MGCC control infrastructure is covered by the DSO. Characteristic values for each local controller were used, namely $300 €$ for each micro wind generator and PVs local controller, $500 €$ for each 
Table 1

Calculated attributes for the five evaluation criteria defined.

\begin{tabular}{|c|c|c|c|c|c|}
\hline Scenario and alternative & $C_{1}[\mathrm{k} €]$ & $C_{2}$ [years] & $C_{3}[\mathrm{MWh}]$ & $C_{4}[\mathrm{t}]$ & $C_{5}[\mathrm{MWh}]$ \\
\hline$I-A$ & 159.63 & 0 & 0 & 0 & 0 \\
\hline$I-B$ & 264.08 & 3 & 59.83 & 110.21 & 8.9 \\
\hline$I-C$ & 394.25 & 5 & 126.43 & 243.91 & 14.42 \\
\hline$I-D$ & 244.13 & 3 & 277.7 & 2142.97 & 10.6 \\
\hline$I-E$ & 362.4 & 5 & 386.32 & 2581.29 & 16.12 \\
\hline$I-F$ & 271.05 & 4 & 523.78 & 4419.45 & 12.29 \\
\hline$I-G$ & 368.18 & 6 & 648.68 & 5025.39 & 17.81 \\
\hline II - A & 159.63 & 0 & 0 & 0 & 0 \\
\hline II - B & 211.08 & 4 & 147.25 & 249.64 & 8.9 \\
\hline II - C & 284.27 & 5 & 295.15 & 552.99 & 14.42 \\
\hline II - D & 200.29 & 4 & 384.61 & 2382.87 & 10.6 \\
\hline II - E & 275.72 & 6 & 598.08 & 3022.51 & 16.12 \\
\hline II - F & 204.39 & 4 & 593.06 & 4668.25 & 12.29 \\
\hline II - G & 270.54 & 7 & 861.06 & 5561.13 & 17.81 \\
\hline III - A & 159.63 & 0 & 0 & 0 & 0 \\
\hline III - B & 261.33 & 2 & 56.13 & 109.04 & 8.9 \\
\hline III - C & 390.22 & 4 & 127 & 240.67 & 14.42 \\
\hline III - D & 250.93 & 2 & 275.56 & 2089.91 & 10.6 \\
\hline III - E & 339.29 & 3 & 1231.22 & 2303.22 & 16.12 \\
\hline III - F & 240.92 & 2 & 470.61 & 4074.41 & 12.29 \\
\hline III - G & 380.28 & 4 & 628.17 & 4774.84 & 17.81 \\
\hline IV - A & 159.63 & 0 & 0 & 0 & 0 \\
\hline IV - B & 211.58 & 2 & 139.45 & 239.37 & 8.9 \\
\hline IV - C & 257.27 & 4 & 267.68 & 483.29 & 14.42 \\
\hline IV - D & 213.42 & 3 & 392.46 & 1828.15 & 10.6 \\
\hline IV - E & 265.09 & 4 & 540.47 & 2631.51 & 16.12 \\
\hline IV - F & 202.45 & 3 & 592.12 & 3941 & 12.29 \\
\hline IV - G & 248.49 & 4 & 769.76 & 4854.15 & 17.81 \\
\hline
\end{tabular}

MGCC and $100 €$ for each LV load local controller. The cost of implementing the CAMC functionality, related to the MMG optimization procedure, is assumed to be $100,000 €$.

These data were adopted, using the experience of the authors in an on-going industrial project in Portugal - the InovGrid project [8] and is used for the purpose of testing the methodology described in the paper. The average interest rate used is assumed to be $7 \%$ and the time horizon is defined by the total number of years of network investment deferral, presented in Table 1, for each alternative of Fig. 3.

Using the simplest methodology, based on average annual data,

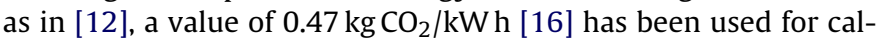
culation of the annual avoided $\mathrm{CO}_{2}$ emissions as a result of the central units' energy replacement by the NC- $\mu \mathrm{G}$ and the CL curtailed using (1-10). This value corresponds to a typical emissions' value of a combined cycle gas turbine running on natural gas, most of the time corresponding to the marginal unit of the Portuguese power system. Assuming that $\mathrm{C}-\mu \mathrm{G}$ units run on natural gas, a reference factor of $0.202 \mathrm{~kg} / \mathrm{kWh} \mathrm{[14]} \mathrm{is} \mathrm{used} \mathrm{to} \mathrm{evaluate} \mathrm{the} \mathrm{annual}$ avoided $\mathrm{CO}_{2}$ emissions of these units for the part related with the electricity production only. In this regard, the emissions avoided to produce electricity are considered to be $1 / 3$ of the total emissions of a microturbine, assuming that the other $2 / 3$ is allocated for heat production. The reliability improvement is assessed assuming

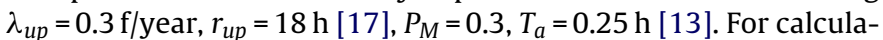
tion of the annual average power generated by the $\mathrm{NC}-\mu \mathrm{G}$, capacity factor of $40 \%$ is used for the wind turbines and $15 \%$ for the PVs [4].

\subsection{Scenarios and alternatives characterization}

In order to evaluate the benefits that may result from the MMG adoption, four mutually exclusive scenarios are defined (Fig. 3) concerning uncertainties due to: (1) Expected annual Load Growth (2\% and 3\% Load Growth-LG); (2) Wholesale Market Prices (Low Market Prices - LMP and High Market Prices - HMP). Seven alternatives (one being the base and six other found credible to reflect different possible MMG concept deployments) are illustrated in Fig. 3(A)- $(G)$, each one presenting a certain amount of NC- $\mu \mathrm{G}, \mathrm{C}-\mu \mathrm{G}$ and CL.

\section{Results}

This section is organized as follows. First, the results concerning the application of the CAMC functionality, described in Section 2.2, are presented. Further on, the impact of the MG and MMG concept is evaluated, where the benefits and costs due to these concepts deployment are modelled within a multicriteria decision aid framework.

\subsection{Application of CAMC functionality}

The results depicted below correspond to a typical winter day in the last year when $\mathrm{C}-\mu \mathrm{G}$ dispatch and $\mathrm{CL}$ shedding under MGCC and CAMC may account for a year of network investment deferral. Figs. 4 and 5 show the outcome of the active network control and management (under CAMC) in LMP period. A slight reduction in the LV active demand and MV branch overloads is detected during daily hours due to NC- $\mu \mathrm{G}$ units' production.

Moreover, typical days of HMP would yield $C-\mu \mathrm{G}$ dispatch and CL shedding for some time intervals (under MGCC), and contribute for a set of indirect benefits to the DSO.

The dispatch of $C-\mu G$ units together with $C L$ shifting, under MGCC, at hour 20 and 21 (Fig. 6) may lead to partial or total resolution of the technical constraint violation due to overloads and the DSO can get the benefit for these hours without having to perform (1-10). However, high branch overload still persists for hour 19, and therefore, (1-10) needs to be performed, potentially leading to an additional year of network upgrade deferral (Fig. 7).

Increased levels of NC- $\mu \mathrm{G}$ may cause technical problems in terms of voltage raise at the LV grid [3]. Therefore, large scale 


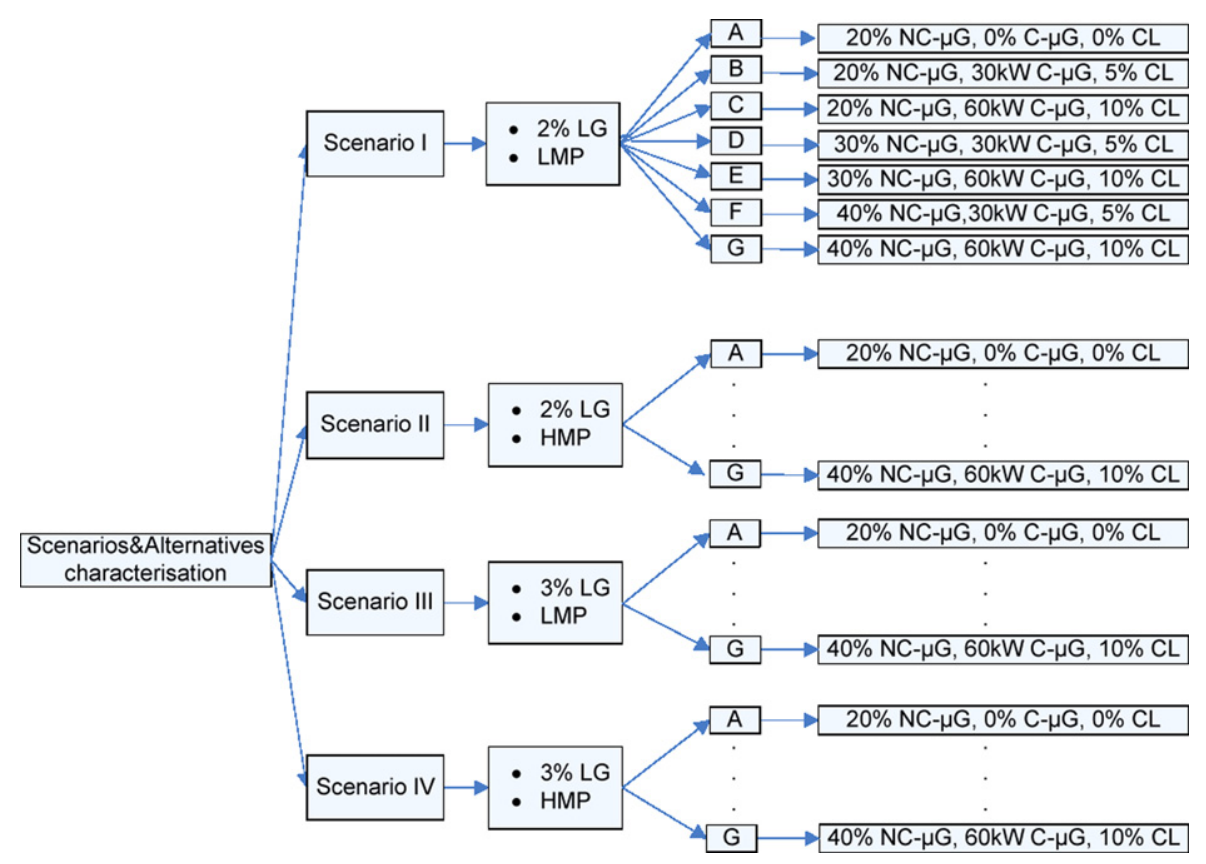

Fig. 3. Scenarios and alternatives characterization.

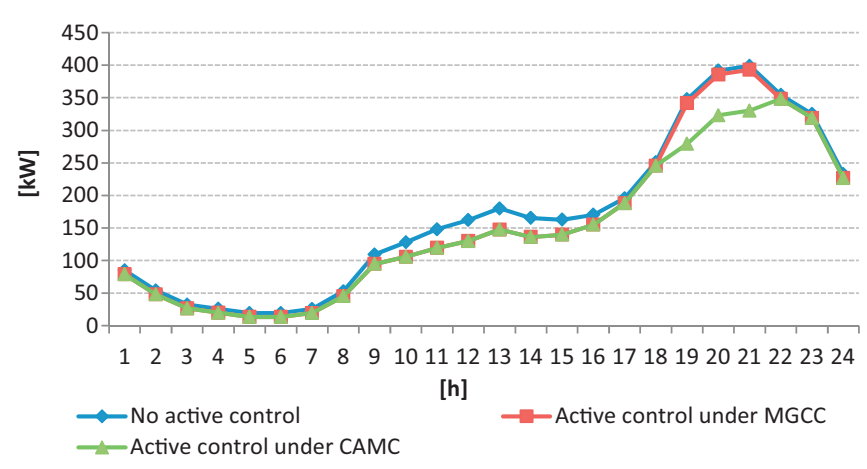

Fig. 4. A single MG demand during LMP period.

integration of $\mu \mathrm{G}$ requires adoption of different functionalities at different MMG control levels. However, this is not the case in our analysis, due to annual load growth going along with the annual growth of $\mu \mathrm{G}$ capacity.

\subsection{Multicriteria assessment}

The multicriteria assessment in this paper shows two sources of complexity in the decision making: (1) multiple criteria of

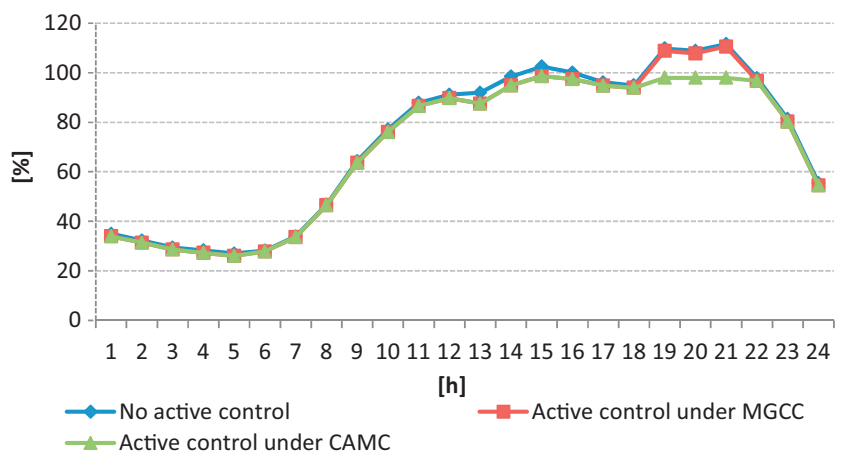

Fig. 5. Load level of the most MV loaded branch during LMP period.

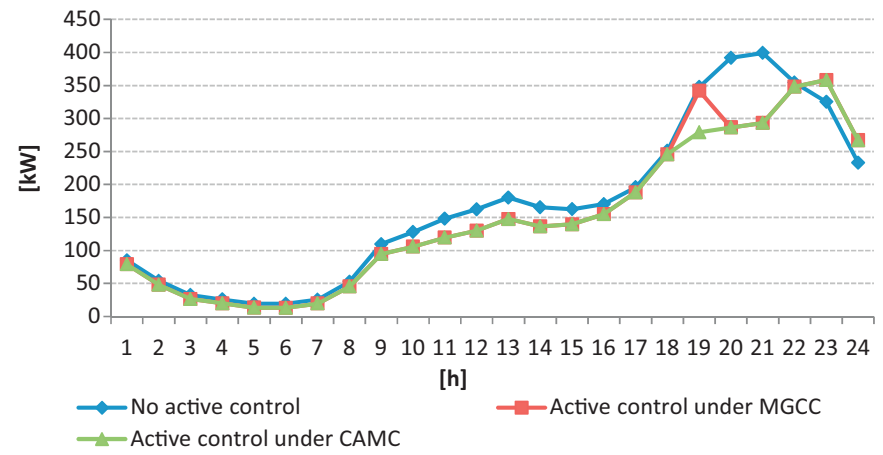

Fig. 6. A single MG demand during HMP period.

evaluation $\left(C_{1}, C_{2}, C_{3}, C_{4}, C_{5}\right)$ to address relevant economic, environmental and technical aspects of the control functionality (1)-(10) under the MMG concept and (2) multiple scenarios to capture uncertainty (4 scenarios) due to load growth and system cost of electricity. The seven alternatives, described in Section 4 , are analysed in a multiattribute framework in order to get an insight into the different aspects of the impact that the DSO may face when dealing with growing $\mu \mathrm{G}$ levels. Once the preferred level of $\mu \mathrm{G}$ penetration and demand side integration is determined, it is then up to

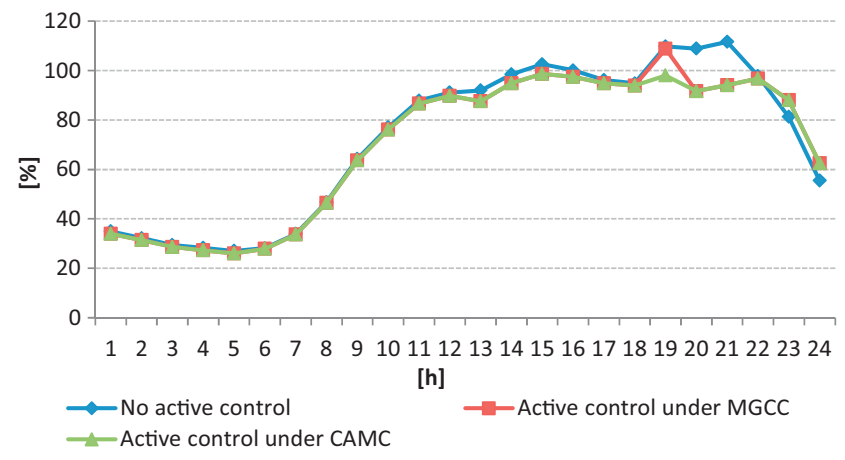

Fig. 7. Load level of the most MV loaded branch during HMP period. 
the DSO to develop adequate incentive and price mechanisms that will lead to such $\mu \mathrm{G}$ and load integration levels. Moreover, other agents (MG consumers, $\mu \mathrm{G}$ developers) may also find the MMG control approach beneficial, where adequate cost/benefit allocation mechanisms should be explored. However, this aspect is beyond the scope of this paper.

Our strategy will be first to deal with the multicriteria problem by conducting the evaluation through trade-off analysis and then capture the uncertainty issue through analysis of regret or disappointment.

As it can be seen from Table 1, alternative A prevails against the other alternatives in the cost criterion. In general, lower costs can be detected during HMP periods (Scenarios II and IV) due to lower operation cost, since $C-\mu G$ units have been dispatched and CL shifted for some hours, under MGCC, as a result of HMP. For the rest of the criteria, alternative $G$, with highest integration of $\mu \mathrm{G}$ and $\mathrm{CL}$ wins over the other alternatives, in each scenario.

\section{Decision aid}

\subsection{Trade-off analysis}

The methodology behind the multicriteria framework used in this research considers trade-off analysis as Decision Aid technique, by defining trade-offs, chosen by the decision maker (the DSO in this study) after careful examination of the situation. Each tradeoff reflects the ratio of improvement in one criterion (for instance, investment deferral) over degradation in another (MMG total cost). Four trade-offs have been defined for each scenario, namely: $\alpha_{1}$ [€/year], which defines the amount of money the DSO is willing to spend in order to have the network upgrade deferred by one year; $\alpha_{2}[€ / \mathrm{MWh}$ ], showing the amount of money paid by the DSO for having the total active losses decreased by $1 \mathrm{MWh} ; \alpha_{3}[€ / \mathrm{t}]$, presenting the willingness of the DSO to pay in order to have $1 \mathrm{t}$ of $\mathrm{CO}_{2}$ emissions decreased, and $\alpha_{4}$ [€/MWh], illustrating the value of $1 \mathrm{MWh}$ avoided NDE due to NC- $\mu \mathrm{G}$ and $\mathrm{C}-\mu \mathrm{G}$ operated under the MG and MMG concepts.

The trade-off values reflect the DSO preferences. For instance, $\alpha_{2}$ reflects the cost of losses increased by the desire of the decision maker to invest in more efficient network in order to obtain an incentive due to loss reduction. The value of $\alpha_{3}$ is related with the $\mathrm{CO}_{2}$ price in the $\mathrm{CO}_{2}$ emission trading market. The revenue attributed to the DSO, due to the energy produced internally within each MG capable to operated isolated from the upstream network that otherwise would be lost in case of upstream network fault, is expressed by $\alpha_{4}$. When evaluating the investment deferral tradeoff $\alpha_{1}$, one may argue that different MV network assets may have different costs. However, we assume that the average cost of MV branch is not substantially different from the average cost of MV/LV transformer. Furthermore, $\alpha_{1}$ depends from the present status of the network and the conditions for its reinforcement. For instance, in highly urban areas, due to a city infrastructure, the MG and MMG concepts deployment may be seen as an only feasible solution of
Table 2

Trade-off analysis and equivalent cost $[\mathrm{k} €$ ] for each alternative and scenario.

\begin{tabular}{lcccc}
\hline Alternative & Scenario I & Scenario II & Scenario III & Scenario IV \\
\hline A & 159.63 & 159.63 & 159.63 & 159.63 \\
B & 240.46 & 171.27 & 243.03 & 182.71 \\
C & 350.95 & 218.2 & 351.98 & 200.49 \\
D & 141.99 & 77.4 & 155.55 & 111.55 \\
E & 228.09 & 106.23 & 155.73 & 121.94 \\
F & 75.83 & -3.84 & 70.3 & 21.11 \\
G & 134.45 & -1.25 & 165.71 & 20.21 \\
\hline
\end{tabular}

the network investment (reinforcement). In that case, $\alpha_{1}$ tends to infinite value.

Starting with indicative values for the 4 trade-offs, such as: $\alpha_{1}=5000$ [€/year], $\alpha_{2}=80$ [€/MWh], $\alpha_{3}=30$ [€/t] and $\alpha_{4}=60$ [€/MW $\mathrm{h}$ ], the equivalent cost is calculated, using (13).

EquivalentCost $=C_{1}-\alpha_{1} \cdot C_{2}-\alpha_{2} \cdot C_{3}-\alpha_{3} \cdot C_{4}-\alpha_{4} \cdot C_{5}$

$C_{1}, C_{2}, C_{3}, C_{4}$ and $C_{5}$ are the attributes values of each criterion. The minus sign before each trade off indicates that the $C_{2}, C_{3}, C_{4}$ and $C_{5}$ correspond to benefits. Still, the equivalent cost does not consider the costs and benefits that are equal in all the alternatives (e.g., the benefit of serving the loads), so this exercise cannot be treated as a cost-benefit analysis.

Table 2 shows the equivalent cost for each alternative in each scenario and above indicated trade-offs. In this case, the alternatives with higher percentage of $\mu \mathrm{G}$ and $\mathrm{CL}$ are ranked higher in all the scenarios, making the MG and MMG concepts more favourable. Alternative F wins over the other alternatives in Scenarios I, II and III, whereas alternative G has the highest rank in Scenario IV (HMP, $3 \% \mathrm{LG})$.

In order to show the flexibility of the methodology to capture decision maker preferences we will consider now a different decision maker that values the investment deferral higher than the previous one (e.g. $\alpha_{1}=10,000$ [ $€ /$ year]). The equivalent cost values would change, favouring alternatives with higher percentage of $\mu \mathrm{G}$ in all the scenarios. For instance, in Scenario II alternative $G$ would now be ranked higher than alternative $F$ and alternative $E$ would now gain over D in Scenario III. This shows that the conclusions depend strongly on the decision maker's preferences expressed by the trade-offs. If necessary, other MCDA techniques, such as value function approach can be used, in order to capture more complex decision maker's preference structures [18].

\subsection{Dealing with uncertainties}

The results shown in Table 2 are very clear about the superiority (given the accepted trade-offs) of alternatives F and G. However, we see that there is a variation of the equivalent cost according to the scenarios, and it may happen that an alternative prevails in some scenarios while being beaten in others. We will illustrate how to deal with that situation, even if in this case the results are more or less obvious. We use the concept of regret or disappointment of a decision and rank the alternatives by minimization of the maximum regret. The idea is to favour alternatives that avoid unpleasant

Table 3

Regret analysis for the equivalent cost $[\mathrm{k} €]$.

\begin{tabular}{|c|c|c|c|c|c|}
\hline Alternative & Scenario I & Scenario II & Scenario III & Scenario IV & Min. max regret rank \\
\hline A & 83.79 & 163.46 & 89.33 & 139.41 & 5 \\
\hline B & 164.62 & 175.11 & 172.73 & 162.49 & 6 \\
\hline C & 275.12 & 222.04 & 281.68 & 180.27 & 7 \\
\hline $\mathrm{D}$ & 66.16 & 81.23 & 85.25 & 91.33 & 2 \\
\hline $\mathrm{E}$ & 152.26 & 110.07 & 85.43 & 101.73 & 4 \\
\hline $\mathrm{F}$ & 0 & 0 & 0 & 0.9 & 1 \\
\hline G & 58.62 & 2.59 & 95.41 & 0 & 3 \\
\hline
\end{tabular}


outcomes in adverse scenarios, taking into account the best that could be achieved in those scenarios.

Operationally, the best value in each scenario $s$ (Table 2 ) has been selected, designated as EquivalentCost* $(s)$ and the regret is calculated, for alternative $z$ due to scenario $s$ :

$\operatorname{Regret}(z, s)=$ EquivalentCost $(z, s)-$ EquivalentCost* $(s)$

Table 3 shows the regret values. The maximum regret of each alternative over the four scenarios is depicted in bold and the alternatives are ranked due to the minimum of the maximum regret experienced. It is easy to confirm that alternative $\mathrm{F}$ gains over the other alternatives, but it is interesting to see that $\mathrm{G}$ is not the second best, due to its very bad result in Sc. III, even if it is the best option in Sc. IV and very good also in Sc. II. However, F, D and G show global superiority regarding the other alternatives.

\section{Conclusions}

This paper deals with the adoption of the MMG concept, as a potential way to facilitate large scale integration of microgeneration. $\mathrm{NC}-\mu \mathrm{G}$ and $\mathrm{C}-\mu \mathrm{G}$ units together with $\mathrm{CL}$ can be actively managed at different control levels, corresponding to different objectives, in normal and stressed MV network operating conditions. For the purpose of dealing with periods of technical constraint violations, such as branch overloads and/or high voltage drops, a new functionality is proposed to be installed at the HV/MV substation level.

Such functionality helps manage the MV distribution network in stressed operating periods by sending requests for CL curtailment and/or C- $\mu \mathrm{G}$ dispatch within each MG. A set of direct benefits, may therefore be attributed to the DSO. Among these benefits one should stress the postponement of grid reinforcements due to expected load growth, especially when dealing with situations of more expensive and sometimes infeasible network upgrades. In addition, HMP yield $C-\mu G$ dispatch and $C L$ shifting at each MGCC control level (in the local retail market) in normal and stressed MV operating hours. These benefits can be attributed to the DSO, assigned as indirect benefits.

Multicriteria analysis is used to highlight that decisions based only on cost analysis do not capture relevant aspects of the problem. Trade off aggregates the different evaluation criteria in a way that helps the decision maker understand the influence of each aspect in the final evaluation. Uncertainties are kept through the creation of four mutually exclusive scenarios that are further analysed using the regret concept, where the decision maker aims to identify the solution that has the best worst-case deviation from the minimum equivalent cost in each scenario (least regret). In this context, alternatives with highest $\mu \mathrm{G}$ penetration and demand side integration outperform over the other alternatives, making the multi microgrid concept preferred solution while less amount of CL and $\mu \mathrm{G}$ does not require adoption of such advanced network concepts.

The analysis performed, considers cost and benefits of developing this approach. Undoubtedly, some of the identified benefits are shared by the MMG consumers. We have assumed an equal share of MMG installation costs between the MMG consumers and the DSO. However, different installation cost sharing mechanisms needs to be further studied.

\section{References}

[1] MORE MICROGRIDS, Advanced Architecture and Control Concepts for More Microgrids, Definition of Ancillary Services and Short-Term Energy Markets, http://www.microgrids.eu/documents/686.pdf.

[2] N. José Gil, J.A. Peças Lopes, Hierarchical frequency and control scheme for islanded Multi Micro-Grid operation, in: Proc. IEEE Power Tech'07 Conference, Lausanne, 2007, pp. 473-478.

[3] A.G. Madureira, et al., Advanced control and management functionalities for multi-microgrids, European Transactions on Electrical Power (2011) 1159-1177.

[4] A.G. Tsikalakis, N.D. Hatziargyriou, Centralized control for optimizing microgrid operation, IEEE Transactions on Energy Conversion 23 (2008) 241-248.

[5] J. Vasiljevska, J.A. Peças Lopes, M.A. Matos, Multi-microgrid impact assessment using multi criteria decision aid methods, in: IEEE Powertech, Bucharest, Romania, 2009, pp. 1-8.

[6] MORE MICROGRIDS, Advanced Architecture and Control Concepts for More Microgrids, Multi-criteria Assessment of Business Cases for Microgrids, http://www.microgrids.eu/documents/683.pdf.

[7] S.S. Rao, Engineering Optimization, Theory and Practice, Wiley, New York, 1996.

[8] L.V. da Cunha, J.P. Lopes, J. Antunes, F. Gomes, J.C. Reis, INOVGRID PROJECT Distribution Network Evolution as a Decisive Answer to New Electrical Sector Challenges, CIRED 2008: Smart Grids for Distribution.

[9] N.D. Hatziargyriou, et al., Quantification of economic, environmental and operational benefits due to significant penetration of microgrids in a typical LV and MV Greek network, European Transactions on Electrical Power (2011) 1217-1237.

[10] H.A. Gil, G. Joos, On the quantification of the network capacity deferral value of distributed generation, IEEE Transactions on Power Systems (2006) 1592-1599.

[11] D. Wang, L.F. Ochoa, G.P. Harrison, C.J. Dent, A.R. Wallace, DG impact on investment deferral: network planning and security of supply, IEEE Transactions on Power Systems (2010) 1134-1141.

[12] A.G. Tsikalakis, N.D. Hatziargyriou, Environmental benefits of distributed generation with and without emissions trading, International Journal on Energy Policy (2007) 3395-3409.

[13] P.M. Costa, M.A. Matos, Regulation of microgeneration and microgrids, International Journal on Energy Policy (2008) 3893-3904.

[14] Portuguese Energy Regulatory Agency, www.erse.pt.

[15] Decree Law of the Portuguese Republic, http://dre.pt/pdf1sdip/2007/11/ 21100/0797807984.PDF (in Portuguese).

[16] Decree Law of the Portuguese Republic, http://dre.pt/pdf1sdip/2008/01/ 01400/0061800619.PDF (in Portuguese).

[17] R.N. Allan, R. Billinton, I. Sjarief, L. Goel, K.S. So, Reliability test system for educational purposes - basic distribution system data and results, IEEE Transactions on Power Systems (1991) 813-820.

[18] R.L. Keeney, Value Focused Thinking, third ed., Harvard University Press, London, 1998 . 